\title{
The hydrodynamic footprint of a benthic, sedentary fish in unidirectional flow
}

\author{
Sheryl Coombs ${ }^{a)}$ \\ Department of Biological Sciences and J.P. Scott Center for Neuroscience, Mind and Behavior, \\ Bowling Green State University, Bowling Green, Ohio 43402

\begin{abstract}
Erik Anderson
Department of Organismic and Evolutionary Biology, Harvard University, Cambridge, Massachusetts 02138 and Department of Applied Ocean Physics and Engineering, Woods Hole Oceanographic Institution, Woods Hole, Massachusetts 02543
\end{abstract}

Christopher B. Braun

Department of Psychology, Hunter College, New York 10021

Mark Grosenbaugh

Department of Applied Ocean Physics and Engineering, Woods Hole Oceanographic Institution, Woods Hole, Massachusetts 02543

(Received 12 July 2006; revised 21 May 2007; accepted 22 May 2007)

\begin{abstract}
Mottled sculpin (Cottus bairdi) are small, benthic fish that avoid being swept downstream by orienting their bodies upstream and extending their large pectoral fins laterally to generate negative lift. Digital particle image velocimetry was used to determine the effects of these behaviors on the spatial and temporal characteristics of the near-body flow field as a function of current velocity. Flow around the fish's head was typical for that around the leading end of a rigid body. Flow separated around the edges of pectoral fin, forming a wake similar to that observed for a flat plate perpendicular to the flow. A recirculation region formed behind the pectoral fin and extended caudally along the trunk to the approximate position of the caudal peduncle. In this region, the time-averaged velocity was approximately one order of magnitude lower than that in the freestream region and flow direction varied over time, resembling the periodic shedding of vortices from the edge of a flat plate. These results show that the mottled sculpin pectoral fin significantly alters the ambient flow noise in the vicinity of trunk lateral line sensors, while simultaneously creating a hydrodynamic footprint of the fish's presence that may be detected by the lateral line of nearby fish. () 2007 Acoustical Society of America. [DOI: 10.1121/1.2749455]
\end{abstract}

PACS number(s): 43.80.Ka, 43.80.Lb, 43.80.Nd [MCH]

Pages: $1227-1237$

\section{INTRODUCTION}

The mottled sculpin (Cottus bairdi) is a benthic freshwater scorpaeniform fish that has a dorso-ventrally compressed head with a fusiform body shape very common in current-swept, freshwater and marine habitats (Webb et al., 1996). These negatively buoyant fish can hold position in currents up to at least $12 \mathrm{~cm} / \mathrm{s}(\sim 1.2$ body lengths $/ \mathrm{s})$ without being swept downstream (Webb et al., 1996; Kanter and Coombs, 2003; Coombs and Grossmann, 2006). To prevent downstream displacement, mottled sculpin may theoretically use at least two different behavioral strategies. One is to orient upstream, thus presenting as low of a drag profile as possible to the downstream forces. Mottled sculpin also make substantial use of their broad pectoral fins, typically displayed outward at an angle of $\sim 45-60^{\circ}$ from the body surface, to resist downstream displacement (Webb et al., 1996). That is, when fish are headed upstream in a current, the leading edges of their pectoral fins are angled downwards

\footnotetext{
a) Author to whom correspondence should be addressed. Electronic mail: scoombs@bgnet.bgsu.edu
}

towards the substrate, thereby generating a downward lift force, which holds the fish to the substrate (Wilga and Lauder, 2001).

Recent studies have shown that visually deprived mottled sculpin from both Lake Michigan and Appalachian stream populations exhibit positive rheotaxis that increases in vector strength with increasing current velocity (Kanter and Coombs, 2003; Coombs and Grossmann, 2006). In the absence of vision, rheotaxis is likely mediated by the superficial neuromasts of the flow-sensing lateral line, which can theoretically determine both the magnitude and direction of the surrounding current (Montgomery et al., 1997, Baker and Montgomery, 1999a,b). In contrast, Lake Michigan mottled sculpin require lateral line canal, but not superficial neuromasts to detect nearby $(\sim 1 / 2$ body length away) artificial prey $(50 \mathrm{~Hz}$ vibrating sphere) (Coombs et al., 2001) and they can readily detect this artificial prey in both still and running water up to current velocities of $8 \mathrm{~cm} / \mathrm{s}$, with only a modest decrease in sensitivity resulting from ambient flow (Kanter and Coombs, 2003).

Not only do the splayed pectoral fins assist sculpin in holding station, they also alter the spatial and temporal char- 
acteristics of the surrounding current. These alterations could provide a hydrodynamic footprint of the fish's presence that is detectable by nearby predators. They could also influence the information that the animal's own lateral line system receives about the direction or magnitude of ambient currents and the presence and location of other hydrodynamic sources (e.g., prey). Although a number of studies have described near-body flow fields and hydrodynamic trails generated behind active swimming organisms (e.g., Stamhuis and Videler, 1995; Müller et al., 1997; Wolfgang et al., 1999; Hanke et al., 2000; Anderson et al., 2001; Hanke and Bleckmann, 2004), very few have described the passive, near-body hydrodynamic effects caused by the presence of stationary benthic organisms in flowing water (e.g., Wilga and Lauder, 2001).

In this study, we use DPIV (digital particle imaging velocimetry) to determine the spatial and temporal characteristics of flow alterations caused by the body and pectoral fins of Lake Michigan mottled sculpin when orienting upstream at different flow velocities. Flow characterizations were done under experimental conditions identical to those used in rheotactic and prey-orienting studies (Kanter and Coombs, 2003), so that results could be directly related to the measured behavioral abilities of mottled sculpin as a function of flow velocity.

\section{METHODS}

\section{A. Animal care and collection}

Mottled sculpin (Cottus bairdi) $(6-8 \mathrm{~cm}$ in standard length) were collected from Lake Michigan using baited minnow traps placed at depths of 1-4 $\mathrm{m}$ in near-shore waters and transported to the Coastal Research Center at Woods Hole Oceanographic Institution, where they were housed in 10 gal aquaria. Water in both home and experimental flow tanks was de-chlorinated tap water maintained at $15 \pm 2{ }^{\circ} \mathrm{C}$. Fish were hand fed small pieces of squid several times a week. Protocols used in the handling of animals were approved by Loyola University Chicago's Institutional Animal Care and Use Committee, the home institution of S. Coombs during the course of these studies.

\section{B. Experimental setup}

Fish were tested in a long Plexiglas rectangular channel of the same dimensions $(44 \mathrm{~cm} \times 18 \mathrm{~cm} \times 17 \mathrm{~cm})$ and of similar design to that used by Kanter and Coombs (2003). The channel was immersed in a downstream section of a large, recirculating, oval-shaped flume $(7.6 \mathrm{~m}$ long, $76 \mathrm{~cm}$ wide, $30 \mathrm{~cm}$ deep), so that water could run through the channel and parallel to its long axis. Flume currents were driven by a conveyor belt of rotating paddles. Two collimators placed at the upstream end of the test channel helped to reduce turbulence in the flow. Fish were placed in a $27 \mathrm{~cm}$ long $\times 18 \mathrm{~cm}$ wide test section bounded by the second collimator on the upstream side and a mesh screen on the downstream side to prevent the fish from escaping into the larger flume. Freestream average velocities in the tank were measured and calibrated against each other with two techniques: DPIV and a Marsh-McBirney flow meter (Model 2000).

\section{Digital particle image velocimetry (DPIV)}

The flow field around the body of a sedentary, benthic fish heading directly upstream was imaged with DPIV (e.g., Adrian, 1991; Willert and Gharib, 1991). Fluid flow around the fish was illuminated by a 0.5 -mm-thick, horizontal laser sheet, which was imaged from below with a high-resolution $(1008 \times 1012)$ digital camera. The flow field was seeded with nearly neutrally buoyant, light-reflective (silver-coated) glass spheres $10 \mu \mathrm{m}$ in diameter. The elevation of the laser sheet was adjusted to between 4 and $12 \mathrm{~mm}$ above the channel substrate. For the normal resting position of sculpin, an $8-9 \mathrm{~mm}$ elevation is at the approximate level of the trunk lateral line canal. The laser sheet was pulsed as a strobe, synchronized to flash once in each frame acquired by the digital camera precisely recording the frame by frame positions of the illuminated fish and seed particles. Particle velocities were then determined by dividing the distance traveled in successive frames by the time interval $(d t)$ between the laser pulses. Laser sheets were pulsed on and off in pairs and the displacement of the particles over time, as well as any movement of the fish, was imaged with a highresolution, digital video camera. The laser pulses were synchronized with the digital camera in pairs such that the first laser pulse of each pair occurred at the end of one video frame and the second at the beginning of the next; the rate of image pair acquisition $(15 \mathrm{~Hz})$ was thus approximately half that of video frame acquisition $(30 \mathrm{~Hz})$. The time interval $(d t)$ between laser pulse pairs varied according to flow speed, ranging from 8 (highest flow speed) to $24 \mathrm{~ms}$ (lowest flow speed).

\section{Data analysis}

To systematically examine the effects of four different flow speeds on both spatial and temporal variations in the flow field, repeated measurements were analyzed from a single individual, $7.5 \mathrm{~cm}$ in standard length. Although this does not allow us to say anything about individual differences, it does rule out inter-individual differences as a source of spatial and temporal variation. Video frame sequences of flow around the fish were examined frame by frame to ensure that only those free of fish-generated movements (other than opercular motions) were selected for further analysis. Selected sequences ranged in total duration from 1.3 to $6.7 \mathrm{~s}$ (20-100 image pairs), with longer sequences at slower flow rates. Each sequence was long enough for a single freestream particle to travel a downstream distance of at least one fish body length. Cross correlation of successive DPIV images was used to produce a two-dimensional (2D) picture of the velocity field. Velocity fields for sequential imaged pairs were then time averaged to determine the mean flow magnitude and direction over a $30 \times 30$ matrix of points (900 total) in a $10 \times 10 \mathrm{~cm}$ field of view (Figs. 1(a)-1(c)). Vorticity plots, derived from the instantaneous two-dimensional flow field, were also time averaged to yield a measure of the mean vorticity magnitude and rotational direction (counterclockwise positive) at each point in the matrix (Figs. 1(d)-1(f)).

In addition to time-averaged plots, instantaneous regional (i.e., spatial) averages of velocity and vorticity were 

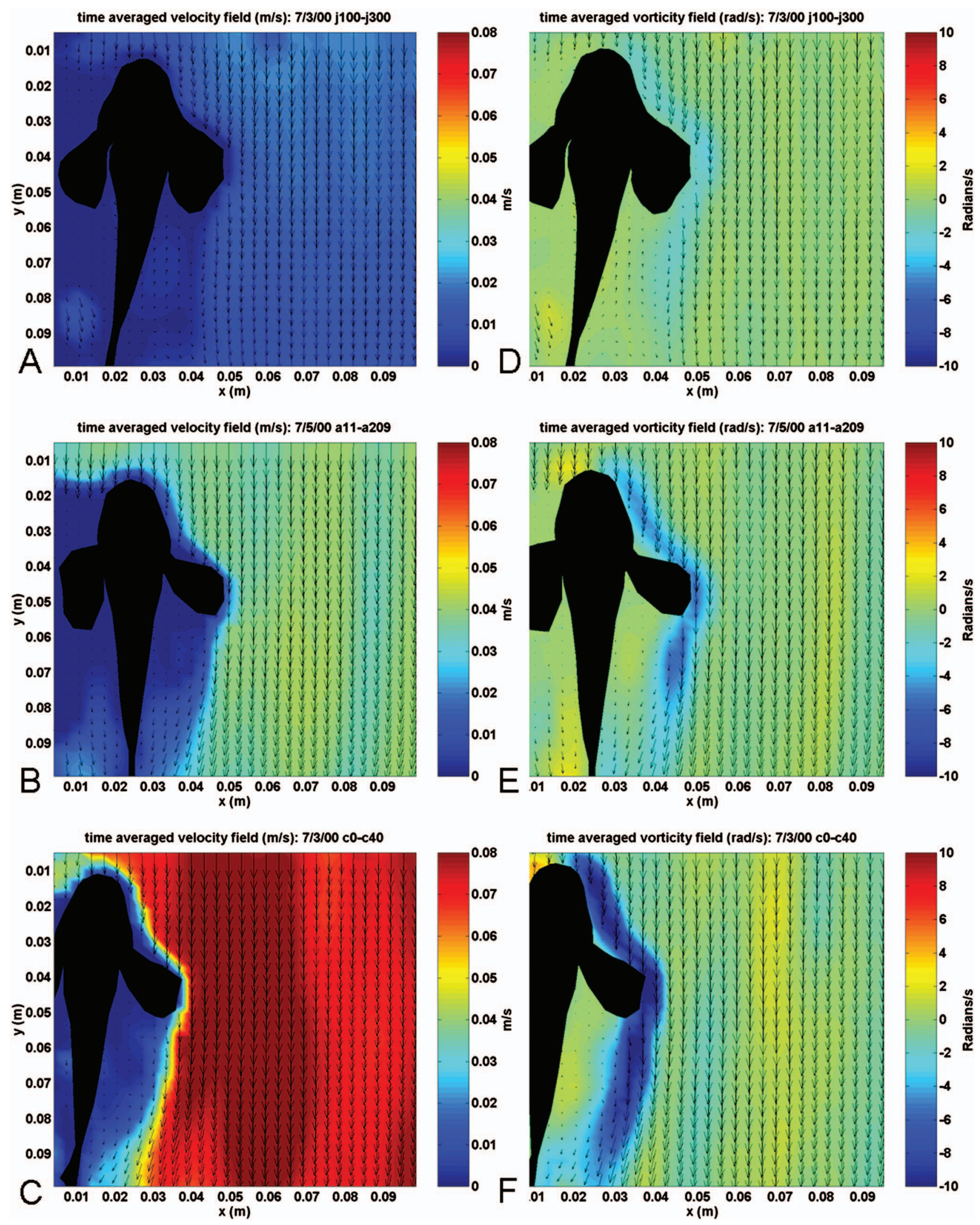

FIG. 1. Time-averaged velocity (a-c) and vorticity (d-e) plots for a single individual at three different background flow levels: $2(\mathrm{a}, \mathrm{d}), 4(\mathrm{~b}, \mathrm{e})$ and $8(\mathrm{c}, \mathrm{f}) \mathrm{cm} / \mathrm{s}$. Laser beam elevation $=8 \mathrm{~mm}$. Arrows depict mean flow directions at a total of 900 different locations in the $10 \times 10 \mathrm{~cm}$ interrogation window and the color spectrum represents mean flow magnitude. Numbers at the upper right-hand corner of each plot indicate the range of frames that were averaged in each case. The average velocity and vorticity plots in a and $\mathrm{d}$, for example, were based on a total of 200 frames (100-300) over a time period of $14 \mathrm{~s}$ $(0.07 \mathrm{~s} /$ frame $\times 200$ frames). The color scale for velocity $(\mathrm{a}-\mathrm{c})$ represents magnitude only, whereas that for vorticity (d-f) represents both magnitude and direction. Clockwise: negative, blue end of spectrum; counterclockwise: positive, red end of spectrum. Note that the light source for illuminating the particles is at the right of the fish and thus, flow information to the left of the fish should be ignored. 



FIG. 2. Time-dependent changes in the signed vorticity (a, b, and c) and vorticity magnitude (d, e, and f) for the following regions of interest: freestream (solid line, open triangles), recirculating (filled squares) and shear layer (open circles) regions. Temporal variations in vorticity are plotted for three different freestream flow rates: $2(\mathrm{a}, \mathrm{d}), 4(\mathrm{~b}, \mathrm{e})$ and $8(\mathrm{c}, \mathrm{f}) \mathrm{cm} / \mathrm{s}$. Vertical lines in each panel indicate the approximate time that it takes for a freestream particle to traverse the length of the fish at different flow velocities.

determined for three specific regions: (1) The freestream region where the flow field was unaffected by the fish's body, (2) a reduced velocity region or separation bubble along the trunk and behind the pectoral fin and (3) the shear layer between the freestream region and the reduced velocity region. Because fish were free to move and their position within the video frame varied, the freestream region was user defined as a $12-16 \mathrm{~cm}^{2}$ rectangular region sufficiently far from the fish ( $>3 \mathrm{~cm}$ away from the fish's midline) and aligned with the velocity vectors and long axis of the fish. The reduced velocity region was defined empirically with a software algorithm that searched a user-defined area $\left(\sim 12 \mathrm{~cm}^{2}\right)$ behind the pectoral fin and along the body surface to determine where the time-averaged velocity fell below a threshold criteria of $0.25 \times$ mean freestream velocity. Similarly, the shear layer was defined by locations within the search region for which the time-averaged vorticity exceeded a threshold criteria of twice the maximum freestream vorticity. Empirically defined recirculating and shear layer regions were then graphed onto 2D, time-averaged velocity and vor- ticity plots and visually inspected to confirm that the algorithms had adequately captured these regions.

Two methods are used to report instantaneous regional vorticity. In the first, we computed the average vorticity, taking into account the sign of the vorticity. The major drawback to this method is that equal amounts of clockwise (-) and counterclockwise $(+)$ vorticity lead to the false conclusion of no vorticity. The advantage of this method is that dominant rotational directions in the vorticity can be revealed when there are unequal amounts of clockwise and counterclockwise rotation. The second method, which avoids the mathematic canceling of counterrotating vorticity, is to simply average the magnitude (i.e., absolute value) of the vorticity in the region. These two methods represent the vector and scalar averages of vorticity, respectively. The vector and scalar averages of vorticity in each of the three regions of interest mentioned above were determined for each image pair of each video sequence and then plotted as a function of time (Fig. 2). Average values over the time series in Fig. 2 were then plotted as a function of the freestream flow veloc- 

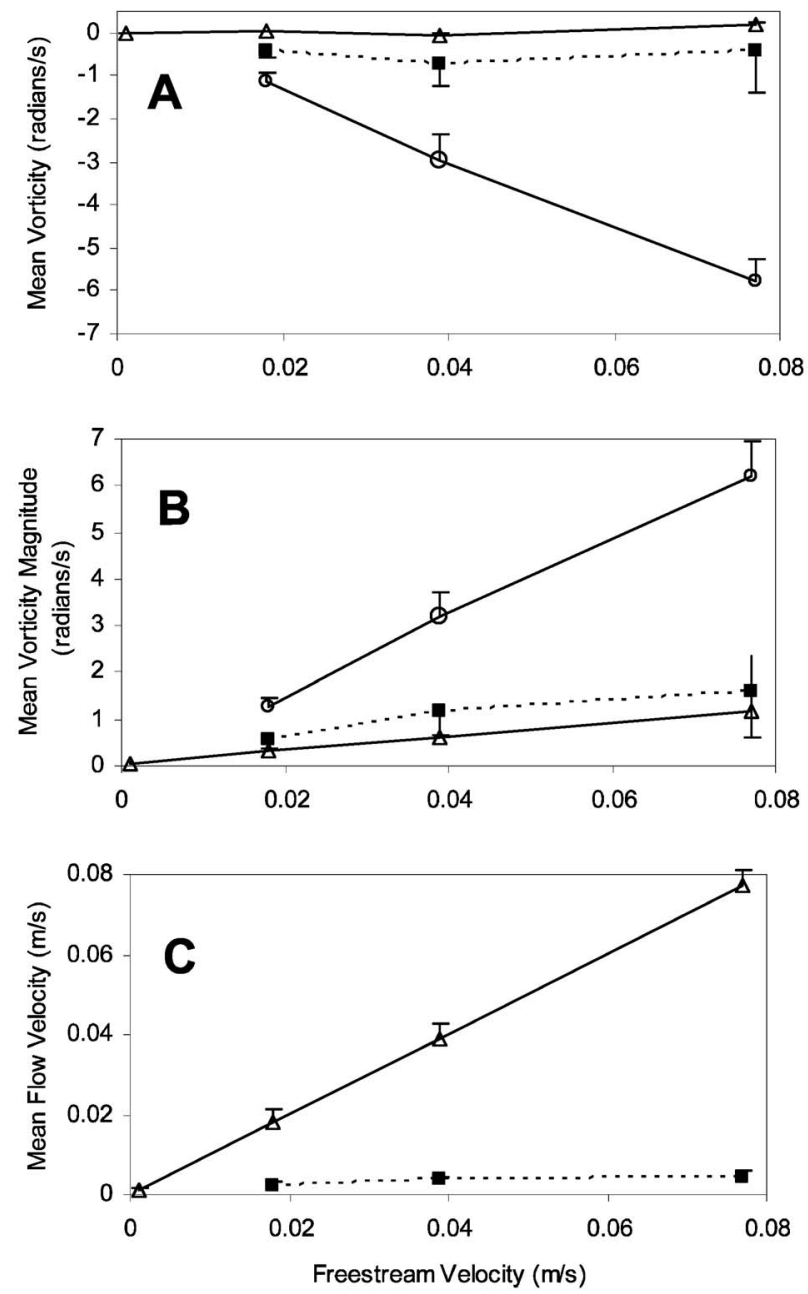

FIG. 3. Time averages of signed vorticities (a), vorticity magnitudes (b) and velocity in the streamwise direction (c) in the separation bubble (filled squares), shear layer (open circles) and freestream (open triangles) regions of the flow field. Instantaneous, spatially averaged values in each region were averaged over time to produce a final mean and standard deviation from the mean. Time averages come from the same data sets used in Fig. 2.

ity (Fig. 3(a)). Likewise, average magnitudes of velocity over time in the three regions of interest were calculated and plotted as a function of freestream flow velocity (Fig. 3(b), magnitude only). Additional time-varying features of the flow field were examined by viewing computer-generated sequences of the instantaneous (1) streamlines and (2) 2D velocity or vorticity plots over time. All measures were calculated using custom routines written in MATLAB (Mathworks, USA).

\section{RESULTS}

\section{A. Regional differences in the flow field}

At sufficient distances from the midline of the fish $(>\sim 3 \mathrm{~cm}$ away), the time-averaged velocity magnitudes and directions were spatially uniform at all flow speeds relative to those closer to the body surface (Figs. 1(a)-1(c)). In this freestream region, the time-averaged vorticity field also revealed some spatial structure, i.e., a tendency for alternating "columns" of clockwise and counterclockwise vortices, presumably due to the presence of the upstream collimator (Figs. 1(d)-1(f)). Nevertheless, clockwise and counterclockwise vortices were nearly equal in abundance, if not randomly distributed relative to near-body regions (Figs. 1(d)-1(f)). This can best be seen from the near-zero values of the signed vorticity, which incorporates both magnitude and rotational direction (sign), both as a function of time for a given velocity (Figs. 2(a)-2(c), open triangles) and as time averages for different velocities (Fig. 3(a), open triangles). In contrast, the time averaged magnitude of vorticity increased with increasing flow velocity (Figs. 1(d)-1(f) and Fig. 3(b), open triangles).

Within $1-2 \mathrm{~cm}$ of the body surface, freestream flow rates as low as $2 \mathrm{~cm} / \mathrm{s}(\sim 0.25$ body lengths $/ \mathrm{s})$ produced significant spatial and temporal nonuniformities in the flow field, including a reduced velocity region or separation bubble behind the pectoral fin (Figs. 1(a)-1(c)). The timeaveraged, velocity magnitude in this region $(2-8 \mathrm{~mm} / \mathrm{s})$ was approximately tenfold less than that of the freestream region $(2-8 \mathrm{~cm} / \mathrm{s})($ Fig. 3(c)) and the time-averaged vorticity (taking both magnitude and direction into account) revealed a slight bias in the clockwise (negative) direction (Fig. 3(a), solid squares) as would be expected for separated flow behind a flat plate at a high angle of attack to the flow. Separation of the flow at the trailing edge of the large, extended 

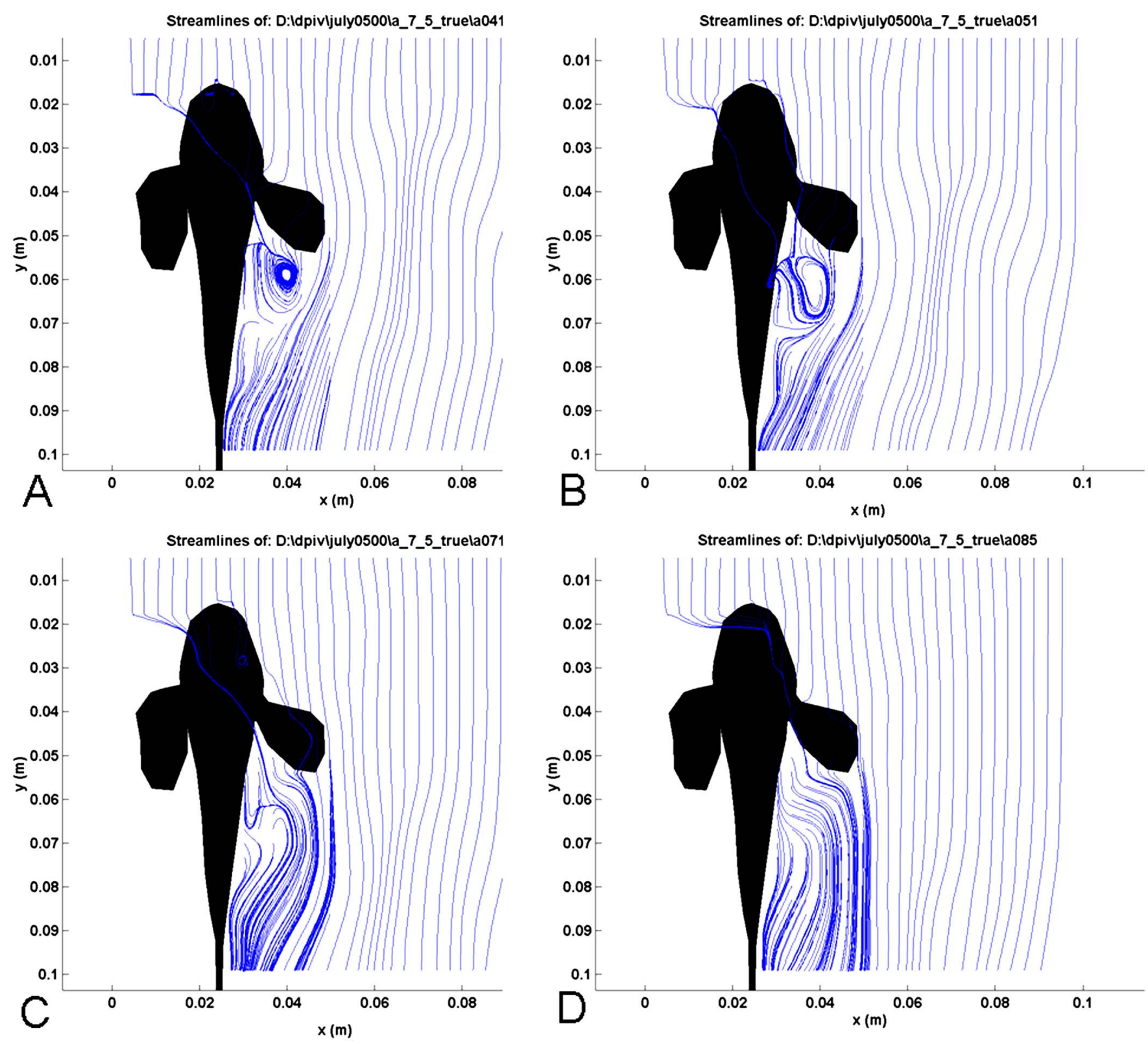

FIG. 4. (Color online) Streamline pictures for the $4 \mathrm{~cm} / \mathrm{s}$ flow condition showing the formation (a) and subsequent shedding (b-d) of a vortex behind the pectoral fin over a time span of approximately $1.5 \mathrm{~s}$. Note that the light source for illuminating the particles is at the right of the fish and, thus, flow information to the left of the fish should be ignored.

pectoral fin and the formation of a separation bubble is clearly revealed in both velocity field (Fig. 1) and streamline plots (Fig. 4). The magnitude of vorticity in the shear layer between the freestream and recirculating regions increased with increasing flow velocities (Figs. 1(d)-1(f), Fig. 3(b), open circles); vorticity in this region also had the expected clockwise (-) rotation (Fig. 1(d)-1(f), Fig. 3(a)).

Closer to the bottom of the test tank (elevation $=4 \mathrm{~mm}$ ) and well within the boundary layer of the substrate, mean flow velocities and vorticity were reduced (Figs. 5(a) and 5(b)) relative to those at the $8 \mathrm{~mm}$ elevation (Figs. 1(c) and 1(f)) for the same freestream velocity $(8 \mathrm{~cm} / \mathrm{s})$.

\section{B. Time-varying changes in the flow field}

Because the DPIV sampling (image-pair acquisition) rate was $15 \mathrm{~Hz}$, we were able to examine temporal changes in the flow field that were slower than $\sim 7.5 \mathrm{~Hz}$, or half the sampling rate. Plotted in Fig. 2 are time series of the spatial averages of the signed vorticity ( $a, b$, and c) and vorticity magnitude ( $\mathrm{d}$, e, and f) for different flow regions and freestream flow rates. As this figure illustrates, the recirculating (filled squares) and shear layer (open circles) zones showed greater temporal oscillations than the freestream region (open triangles) at all flow speeds.

Time-varying changes in these spatially averaged values do not adequately capture all of the temporal and spatial changes that occur within a given region, however. As the flow separates off the pectoral fin, for example, the resulting shear layer boundary between the freestream and the recirculating region exhibits a slow $(<1 \mathrm{~Hz})$, wave-like undulation towards and away from the body surface, as observed from animated film sequences of $2 \mathrm{D}$ vorticity plots. As a 

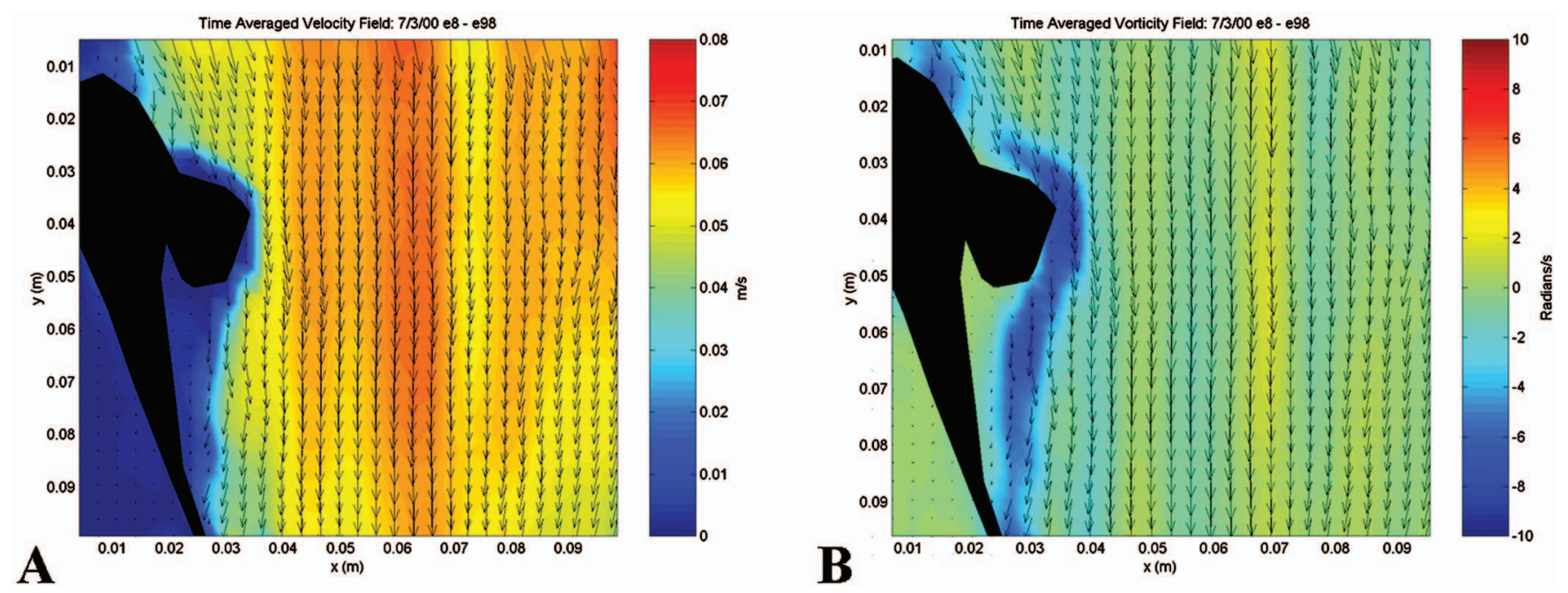

FIG. 5. Time-averaged velocity (a) and corresponding vorticity (b) plots for an $8 \mathrm{~cm} / \mathrm{s}$ flow at a laser beam elevation of $4 \mathrm{~mm}$. Compare with results from $8 \mathrm{~mm}$ beam elevation at same flow speed (Figs. 1(d) and 2(d)). Note that flow information to the left of the fish should be ignored.

result, the size and shape of the recirculating region behind the pectoral fin also changes. These slow fluctuations are most likely due to the periodic shedding of vortices, which form at the trailing edge of the pectoral fin (Figs. 4(a) and 4(b)) and are correlated with flow changes at the fish body surface. After a shedding event, a new vortex forms and the recirculating flow becomes more prominent. Fluid along the body behind the pectoral fin was observed to flow slowly upstream. Further downstream, near the peduncle, the flow was generally streamwise. Periodic vortex shedding adds a highly unsteady series of flow fields which "dilute" steady aspects of the time-averaged flow as the vortex moves downstream. Thus, in plots of time-averaged velocity, the average circulatory flow directly behind the pectoral fin is weaker than one might expect (Figs. 1(a)-1(c)). If the vortex behind the pectoral fin were stable, i.e., not-shedding periodically, the time-averaged velocity plots would reveal a more obvious circulatory flow. As one would expect, the vortex was more stable at slower flows; that is, shedding was less frequent, and indeed the circulatory flow directly behind the pectoral fin in the time-averaged velocity plots is most easily observed in the slower, $2 \mathrm{~cm} / \mathrm{s}$ case (e.g., note upstreamdirected arrows behind the pectoral fin in Fig. 1(d)).

Although not a planned part of this study, we were also able to discern respiratory flows due to the slow motions of the fish's operculum in the absence of imposed flow (Figs. 6(a) and 6(b)). The respiratory flow appeared to consist of a inhalant flow near the mouth and an exhalent flow caudal to the operculum and pectoral fin. The exhalant flow consisted of a slow dc $(\sim 2 \mathrm{~mm} / \mathrm{s})$ component that was ac modulated at the rate of $\sim 0.7 \mathrm{~Hz}$ (Fig. 6(d)). AC fluctuations in surrounding flow velocity were also seen for several centimeters rostral to the pectoral fin, but without much evidence for a significant dc component (Fig. 6(c)). The $p k-p k$ amplitude of ac flow modulations was greater near the trunk $(\sim 2 \mathrm{~mm} / \mathrm{s}$; Fig. 6(d)) than head $(\sim 1 \mathrm{~mm} / \mathrm{s}$; Fig. 6(c)).

\section{Regional differences in signal-to-noise ratios in a prey-detection context}

In order to gain an appreciation for how signal-to-noise $(\mathrm{S} / \mathrm{N})$ ratios might vary with ambient flow conditions in different regions of the flow field, we replotted signal detection data from previous experiments (Kanter and Coombs, 2003) conducted under ambient flow conditions nearly identical to those used in this study. In these experiments, the preyorienting responses of mottled sculpin were used to determine threshold signal levels required for a $50 \%$ probability of orienting towards a small (6 mm diam), artificial prey (a $50 \mathrm{~Hz}$ vibrating sphere). Fish were oriented upstream at the time of signal onset and the signal source was approximately $5 \mathrm{~cm}$ (measured from the centerline) to the side of the fish at about the same rostro-caudal level as the point of pectoral fin insertion.

For the purpose of computing S/N ratios, threshold signal levels at the source (in $p k-p k$ velocity units), rather than at the body surface of the fish, were used because these values do not involve untested assumptions about signal attenuation and presumed levels at the sensory surface of the fish. Noise levels were characterized in terms of mean velocity (as displayed in Fig. 3(c)), using the average values from the time series of spatially averaged magnitudes over the freestream region and reduced velocity (separation bubble) region behind the pectoral fin. $\mathrm{S} / \mathrm{N}$ ratios in $\mathrm{dB}\left(20^{*} \log \right.$ $[\mathrm{S} / \mathrm{N}])$ were then plotted as a function of mean noise level in $\mathrm{dB}$ re: the noise level in no flow conditions (i.e., $20^{*} \log \left[N_{x} / N_{0}\right]$, where $N_{x}=$ noise level for either the $0,2,4$ or $8 \mathrm{~cm} / \mathrm{s}$ flow conditions and $N_{0}=$ noise level for the $0 \mathrm{~cm} / \mathrm{s}$ flow condition) (Fig. 7). Since the no-flow condition did not produce distinct freestream and reduced velocity regions, a single noise level, based on a spatial average in the freestream region (as defined previously), was used to compute $\mathrm{S} / \mathrm{N}$ ratios in the $0 \mathrm{~cm} / \mathrm{s}$ flow condition. The nonzero noise values for this condition are presumably due to substrate vibrations, convective currents and the animal's own respiratory movements (Fig. 6). 

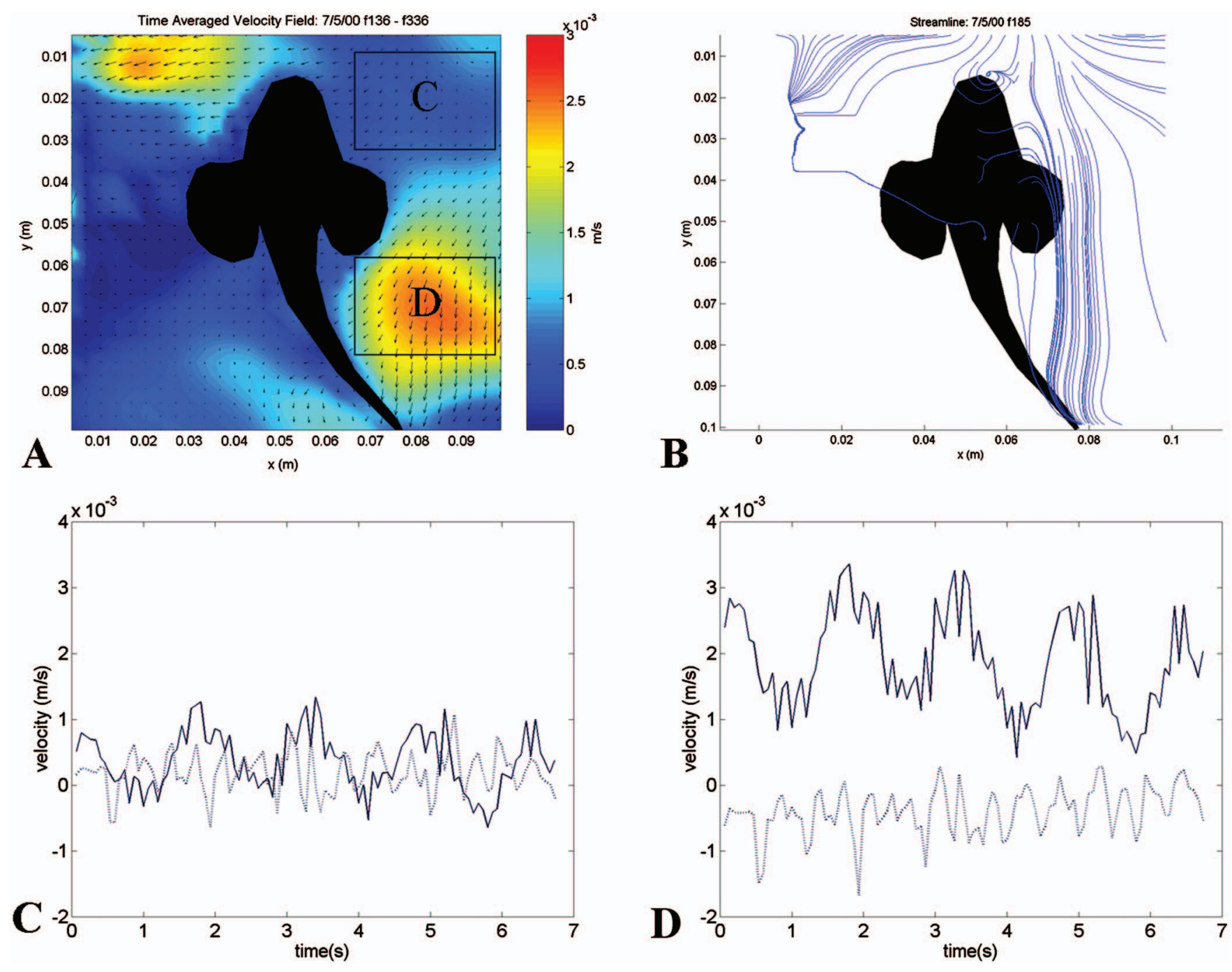

FIG. 6. Time-averaged velocity data are scaled to minimum and maximum velocity levels to show spatial patterns of fish-generated respiratory flow in the absence of imposed flow (a). Corresponding streamlines for a given image pair at a single moment in time are depicted in (b). Low- and high-velocity regions in front of (low) and behind (high) the pectoral fin were spatially averaged for each image pair of the video sequence to show time-varying changes in the mean respiratory flow for each region (low, c; high, d). Solid lines in c and d track spatially averaged velocity magnitudes in the streamwise (parallel to the long axis of the flow tank) direction, whereas dashed-lines track velocity magnitudes in the crosswise direction.

We should also point out that threshold signal levels from behavioral studies were measured within a $3 \mathrm{~Hz}$ bandwidth with an analog wave analyzer centered at $50 \mathrm{~Hz}$. In contrast, DPIV sampling rates limited noise level measurements to frequencies below $\sim 7.5 \mathrm{~Hz}$. Thus, time wave forms of slow ac flows like those produced by the animal's own respiratory movements could be recovered (Figs. 6(c) and 6(d)), but those for higher frequency noises could not. In essence, the $\mathrm{S} / \mathrm{N}$ ratios reported here represent ac signal levels at $50 \mathrm{~Hz}$ relative to low-frequency $(\mathrm{dc}-7 \mathrm{~Hz})$ noise levels. Thus, it is important to point out that they can be nothing more than crude estimates of how S/N ratios vary with location and flow speed.

$\mathrm{S} / \mathrm{N}$ ratios based on behavioral thresholds for the detection of a $50 \mathrm{~Hz}$ signal varied according to the spatial location of the noise (Fig. 7). Theoretical predictions for the complete presence or absence of noise interference (masking) are plotted for comparison (heavy solid lines in Fig. 7). Perfect masking predicts that, e.g., a twofold increase in noise will produce a twofold increase in the minimum level of the sig-

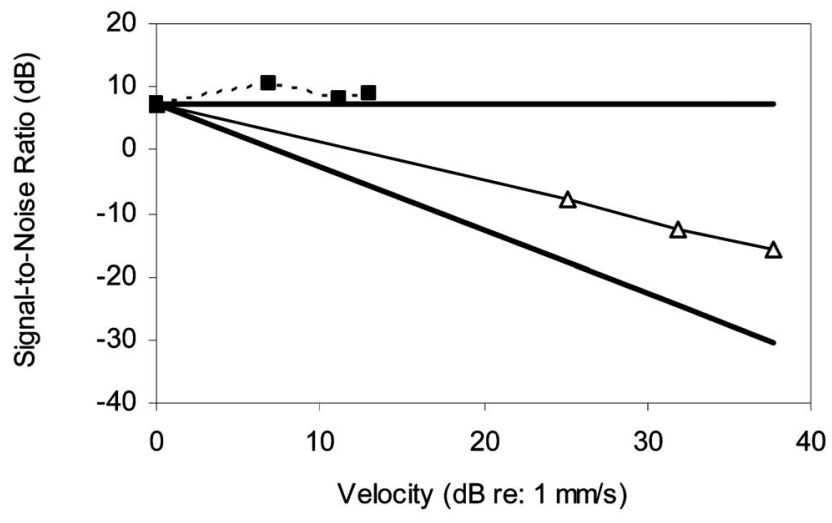

FIG. 7. Signal-to-noise ratio functions for two different regions of the flow field: freestream (solid line with open triangles) and recirculating region behind the pectoral fin (dashed line with solid squares). Thick solid lines show theoretical predictions for the complete presence $($ slope $=0)$ and absence (slope $=-1$ ) of noise interference. See text for further details. Slope, $R$ and $P$-value (slope significantly different from zero) regression statistics for $\mathrm{S} / \mathrm{N}$ functions are $-0.65,0.99,0.00023$ (freestream) and $-0.13,0.77,0.226$ (separation bubble), respectively. 
nal required for detection, resulting in $\mathrm{S} / \mathrm{N}$ ratios that remain constant (solid line with slope of 0 ). A complete absence of masking, on the other hand, predicts that threshold signal levels will stay the same, regardless of ambient noise levels; $\mathrm{S} / \mathrm{N}$ ratios will thus decline by the same amount as the noise level increases (solid line with slope of -1). As Fig. 7 shows, the $\mathrm{S} / \mathrm{N}$ function for the freestream region has a negative slope near -1 that is significantly different from 0 , whereas the $\mathrm{S} / \mathrm{N}$ function for the recirculating region has a slope that is not significantly different from zero (see figure legend for regression statistics).

\section{DISCUSSION}

\section{A. Species comparisons}

Our results show that the large pectoral fin of the mottled sculpin significantly alters ambient currents near the body surface in the vicinity of the lateral line and also behind the fin to leave a hydrodynamic wake. For fish heading upstream, the ambient flow is deflected by the pectoral fin and separates along the fin's edges, resulting in a low-velocity, recirculating region behind the pectoral fin and a trailing wake that presumably travels beyond the length of the fish.

In principle, our results are similar to those obtained by Wilga and Lauder (2001) in their study of pectoral fin function during station holding by the benthic bamboo shark (Chiloscyllium plagiosum). These investigators used DPIV to construct a 2D velocity matrix in the vertical plane for the purpose of computing lift forces on the pectoral fin of bamboo shark during station holding behavior in an upstream direction. Velocity profiles in this plane revealed a wake of clockwise vortices and a region of upstream flow behind and below the dorsal and trailing edge of the pectoral fin similar to those observed in the horizontal plane for the mottled sculpin behind the lateral edge of the pectoral fin and between the fin and the body surface.

In currents varying from 0 to 1 body length/s (up to $\sim 50 \mathrm{~cm} / \mathrm{s}$ ), bamboo sharks were also observed to adopt station-holding behaviors similar to those of the mottled sculpin, including positive rheotaxis and an adjustment of the pectoral fin angle so that the leading edge was increasingly more ventral to the trailing edge as flow velocity increased. Although we did not directly measure fin angle in mottled sculpin as a function of flow velocity, we did observe a change in the pectoral fin position from one that was more nearly horizontal at 0 and $2 \mathrm{~cm} / \mathrm{s}$ flow velocities to one that was more nearly vertical at higher flow velocities, with the leading (upstream) edge being more ventral than the trailing (downstream) edge. This effect can be seen as a corresponding change in the horizontal aspect of the fin, which has been traced from videotape images onto the velocity and vorticity plots (e.g., compare outline of right fin in Figs. 1(a) $(2 \mathrm{~cm} / \mathrm{s})$ and $1(\mathrm{c})(8 \mathrm{~cm} / \mathrm{s}))$. In bamboo sharks, the vertical adjustment of the pectoral fin leads to negative lift forces as high as $-0.084 \mathrm{~N}$ for a flow velocity of 0.75 body lengths/s (Wilga and Lauder, 2001). Thus, it is quite likely that pectoral fin positioning by mottled sculpin results in negative lift as well, as previously hypothesized by Webb et al. (1996). The friction of the animal against the substrate due to these negative lift forces helps fish to maintain a stationary position. Downstream drag forces, which result from high pressure areas in front of the fish and low pressure areas in the low-velocity, recirculating region behind the pectoral fin, and fluid shear in the boundary layer along the fish surface (Anderson et al., 2001), work against this friction. Should these drag forces exceed the friction with the substrate, the fish will lose position.

Although it is tempting to speculate that species-specific differences in body form (e.g., the shape of the body or the shape, size, and insertion of the pectoral fin) might lead to quantitative or qualitative differences in the hydrodynamic signatures of these two species, this proposition is difficult to evaluate based on these two studies alone. For an equivalent flow speed of $\frac{1}{2}$ body lengths/s, the maximum vorticity magnitude in the wake of the bamboo shark's pectoral fin ( $\sim 5$ radians/s @ 17 cm/s; Wilga and Lauder, personal communication) was considerably higher than that measured for the mottled sculpin ( $\sim 6$ radians/s @ 4 cm/s) (Fig. 1(e)). How much of this difference is due to (1) morphological or kinematic differences between the two species, (2) differences in absolute flow speeds or (3) methodological differences in the plane of measurement or the exact location of the plane relative to the fish remains to be seen. Our measurements in the horizontal plane at two elevations (4 and $8 \mathrm{~mm}$ ) (compare Figs. 1 and 5) clearly show that elevation can make a dramatic difference, not only in terms of vorticity magnitudes in the wake, but also in terms of vorticity and velocity magnitudes in the freestream region. Future DPIV studies should address all of these factors, and should include measurements taken at different elevations.

The wake behind the pectoral fin of the benthic sculpin is potentially a rich source of information to other nearby fishes. Although wakes actively generated by moving appendages and swimming fish have been widely studied (e.g., Hanke and Bleckmann, 2004), those generated by the passive interaction of an appendage in flowing water is less widely studied and appreciated (Fish and Lauder, 2006). Nevertheless, both behavioral (Pohlmann et al., 2001, 2004) and physiological (Chagnaud et al., 2006) experiments have shown that actively generated wakes and their vortex structures are potent lateral line stimuli. Catfishes preying on guppies are able to follow the wake left behind a swimming guppy for several seconds (up to $10 \mathrm{~s}$ ) and at substantial distances from the prey (Pohlmann et al., 2001; 2004). Moreover, primary lateral line afferents can code information about the size, shape and rotational direction of passing vortices in the wake of a stationary cylinder in a stream (Chagnaud et al. (2006)). It is likely that fish can analyze the hydrodynamic structure of a wake to determine the producer's size, swimming speed, mode of locomotion, and perhaps more. Future research should provide greater characterization of the hydrodynamic structures present in both passively and actively generated fish wakes and use similar structures in behavioral and physiological experiments to determine if and how fish make use of this hydrodynamic information. 


\section{B. Respiratory signals}

It is well known that the ac modulation of dc electric currents across the gill epithelia of fish produce weak bio electric signals that can be detected by the electrosensory systems of nearby hetero- (e.g., predators) or conspecifics in the context of prey acquisition and mating behaviors (Sisneros and Tricas, 2002). In contrast, respiratory flow as a biologically relevant signal detected by the mechanosensory lateral line has received far less attention. For mottled sculpin, the nearby $(<3 \mathrm{~cm}$ away from the fish) respiratory flow, which is amplitude modulated at the frequency $(f)$ of $0.7 \mathrm{~Hz}$, is relatively weak $\sim 2 \mathrm{~mm} / \mathrm{s}$ ( $p k-p k$ velocity, $u$ ) or $\sim 9 \mathrm{~mm} / \mathrm{s}^{2}$ (pk-pk acceleration, $a$, where $a=2$ pi $f u$ ). Nevertheless, these levels are above threshold velocity and acceleration levels of response $\left(\sim 0.01 \mathrm{~m} / \mathrm{s}\right.$ and $1 \mathrm{~mm} / \mathrm{s}^{2}$, respectively) for mottled sculpin lateral line nerve fibers (Coombs and Janssen, 1990).

According to Hughes and Morgan (1973), a continuous, anterior-to-posterior flow of water through the mouth and across the gills in nonram ventilating fishes involves two pumps that are phase locked to alternately push (pressure pump) and pull (suction pump) water across the gills from the oropharyngeal to the parabranchial cavity. Our DPIV results are consistent with this description in that we see a continuous, downstream flow (Fig. 6(b)) that is ac modulated by gill movements (Figs. 6(c) and 6(d)). The modulation is strongest behind the operculum and pectoral fin (Figs. 6(a) and 6(d)), but weaker modulations are also observed frontally and laterally (Figs. 6(a) and 6(c)). It is worth pointing out that the pumping action of the opercula also changes the size and shape of the head. Thus, if sculpin maintain active branchial ventilation when exposed to externally imposed flows, changes in opercular positions are likely to cause further alterations in the surrounding (imposed+selfgenerated) flow field. Actively respiring animals may thus be surrounded by a bubble of temporal modulations that could give away their presence, whether ambient currents are present or not.

\section{Effects of regional noise differences on signal detection and processing}

The extent to which local flow alterations impact received information by the lateral line is still largely an open question, but it is likely that the effects will vary, depending on the behavioral task at hand, the location and type of both signal sources and lateral line sensors (i.e., superficial vs. canal neuromasts), the type and character of the ambient noise, and the overall size, shape and position of the fish's body and fins. In still-water conditions, the sensitivity of the mottled sculpin lateral line system to both live (e.g., Daphnia) and artificial (vibrating sphere) prey varies according to prey location. Sensitivity to prey along the trunk is poorer than that to prey along the head, though not directly in front of the head (Hoekstra and Janssen, 1986; Coombs and Janssen, 1990). Regional differences in sensitivity have been correlated with a variety of anatomical differences including the fact that neuromasts on the head are more densely packed and have greater numbers of hair cells per neuromast than those on the trunk (Janssen et al., 1987).

There is presently very little information on how these sensitivity differences might affect prey detection when flow noise is present. Given that the sculpin's body alters the ambient noise field in the vicinity of the lateral line, the signalto-noise ratio is likely to vary at different sensor locations along the body surface. It is conceivable, for example, that the ability of sculpin to detect small epibenthic prey within the reduced velocity region behind the pectoral fin would be nearly equivalent or even enhanced relative to their ability to detect the same prey at an equivalent distance from the head, where noise levels approach those in the freestream region. In any event, it is clear that regional differences in sensitivity need to be reexamined in terms of $\mathrm{S} / \mathrm{N}$ ratios at different body locations.

In the context of prey detection by mottled sculpin in stream conditions, the choice of where flow noise is measured can lead to dramatically different conclusions about signal-to-noise processing capabilities (Fig. 7). For noise levels measured in the recirculating region behind the pectoral fin, $\mathrm{S} / \mathrm{N}$ ratios were largely independent of the noise level, following the theoretical prediction for the presence of noise interference. Taken at face value, this "view" of the results is consistent with the idea that both signal and noise were "passed" through the same, low-pass channel (i.e., the superficial neuromast submodality of the lateral line), thus allowing the noise to interfere with signal detection. In contrast, when $\mathrm{S} / \mathrm{N}$ ratios were based on noise levels measured in the freestream region, $\mathrm{S} / \mathrm{N}$ ratios were seen to decrease with increasing noise levels, following the theoretical prediction for the absence of noise interference. In other words, this "view" of the results leads to a very different explanation-that the noise, but not the signal, was largely rejected by the known, high-pass filtering actions of lateral line canals.

In this particular case, there are several independent lines of evidence to suggest that the latter conclusion is most likely correct (as reviewed in Kanter and Coombs, 2003), including neurophysiological evidence (Engelman et al., 2002) from goldfish showing that superficial neuromast, but not canal neuromast responses to dipole signals are reduced in the presence of flowing water relative to those in still water. Clearly, measurements of both signal and noise levels in different regions adjacent to the lateral line are needed before firm conclusions can be reached on this question. Nevertheless, this exercise illustrates the complexity of the problem and the care that must be taken to reach valid conclusions about $\mathrm{S} / \mathrm{N}$ processing capabilities of the spatially distributed lateral line system.

Ironically, it is usually assumed that lateral line function, in particular that of superficial neuromasts, will be compromised in the presence of ambient flows, but in fact, local alterations in the flow field around the mottled sculpin's body predict that neuromasts behind the pectoral fin and along nearly the entire length of the trunk may not be compromised at all-at least with respect to the detection of nearby prey in this region. This puts a slightly new spin on the old hypothesis that lateral line sensors in some species may have been evolutionarily "rerouted" around the pectoral fin to circum- 
vent self-stimulation by the animal's own fin movements (Dijkgraaf 1963). Indeed, the arching of the trunk canal above the pectoral fin is correlated with the general shape and size of the pectoral fin in a wide range of actively swimming fishes, but this does not appear to be the case for many sedentary, benthic fishes like the mottled sculpin (Webb, 1989). Rather than acting as a constant source of selfstimulation, the huge pectoral fin may in this case actually provide shelter from high levels of ambient flow noise to sensors on the trunk.

\section{ACKNOWLEDGMENTS}

This work was funded in part by an NIDCD program project grant to the Parmly Hearing Institute, Loyola University Chicago (W. Yost, PI, S. Coombs, Co-PI). S.C. and C.B.B. thank R. and K. Fay for their generous hospitality while we conducted this research at WHOI. Funding for the DPIV experiments was provided by NSF Grant No. IBN0114148 and the Woods Hole Oceanographic Institution.

Adrian, R. J. (1991). "Particle imaging techniques for experimental fluid mechanics," Annu. Rev. Fluid Mech. 20, 421-485.

Anderson, E. J., McGillis, W. R., and Grosenbaugh, M. A. (2001). "The boundary layer of swimming fish," J. Exp. Biol. 204, 81-102.

Baker, C. F., and Montgomery, J. C. (1999a). "Lateral line mediated rheotaxis in the Antarctic fish Pagnothenia borchgrevinki," Polar Biol. 21(5), 305-309.

Baker, C. F., and Montgomery, J. C. (1999b). "The sensory basis of rheotaxis in the blind Mexican cave fish, astyanax fasciatus," J. Comp. Physiol. 184, 519-527.

Chagnaud, B. P., Bleckmann, H., and Engelmann, J. (2006). "Neural responses of goldfish lateral line afferents to vortex motions," J. Exp. Biol. 209, 327-342.

Coombs, S., and Grossmann, G. (2006). "Mechanosensory-based orienting behaviors in fluvial and lacustrine populations of mottled sculpin (Cottus bairdi)," Mar. Freshwater Behav. Physiol. 39, 113-130.

Coombs, S., and Janssen, J. (1990). "Behavioral and neurophysiological assessment of lateral line sensitivity in the mottled sculpin, Cottus bairdi," J. Comp. Physiol., A 167, 557-567.

Coombs, S., Braun, C. B., and Donovan, B. (2001). "Orienting response of Lake Michigan mottled sculpin is mediated by canal neuromasts," J. Exp. Biol. 204, 337-348.

Dijkgraaf, S. (1963). "The functioning and significance of the lateral-line organs," Biol. Rev. Cambridge Philos. Soc. 38, 51-105.
Fish, F. E., and Lauder, G. V. (2006). "Passive and active flow control by swimming fishes and mammals," Annu. Rev. Fluid Mech. 38, 193-224.

Hanke, W., Brücker, C., and Bleckmann, H. (2000). "The aging of the lowfrequency water disturbances caused by swimming goldfish and its possible relevance to prey detection,” J. Exp. Biol. 203, 1193-1200.

Hanke, W., and Bleckmann, H. (2004). "The hydrodynamic trails of Lepomis gibbosus (Centrarchidae), Colomesus psittacus (Tetraodontidae) and Thysochromis ansorgii (Cichlidae) investigated with scanning particle image velocimetry,” J. Exp. Biol. 207(9), 1585-1596.

Hoekstra, D., and Janssen, J. (1986). "Lateral line receptivity in the mottled sculpin Cottus bairdi," Copeia 1, 91-96.

Hughes, G. M., and Morgan, J. (1973). "The structure of fish gills in relation to their function," Biol. Rev. Cambridge Philos. Soc. 48, 419-475.

Janssen, J., Coombs, S., Hoekstra, D., and Platt, C. (1987). "Postembryonic growth and anatomy of the lateral line system in the mottled sculpin, Cottus bairdi (Scorpaeniformes: Cottidae)," Brain Behav. Evol. 30, 210229

Kanter, M., and Coombs, S. (2003). "Rheotaxis and prey detection in uniform currents by Lake Michigan mottled sculpin (Cottus bairdi)," J. Exp. Biol. 206, 59-60.

Montgomery, J. C., Baker, C. F., and Carton, A. G. (1997). "The lateral line can mediate rheotaxis in fish," Nature (London) 389, 960-963.

Müller, U. K., van den Heuvel, B. L. E., Stamhuis, E. J., and Videler, J. J. (1997). "Fish footprints: morphology and energetics of the wake behind a continuously swimming mullet (Chelon labrosus Risso)," J. Exp. Biol. 200, 2893-2906.

Pohlmann, K., Grasso, F. W., and Breithaupt, T. (2001). "Tracking wakes: The nocturnal predatory strategy of piscivorous catfish," Proc. Natl. Acad. Sci. U.S.A. 98, 7371-7374.

Sisneros, J. A., and Tricas, T. C. (2002). "Neuroethology and life history adaptations of the elasmobranch electric sense," J. Physiol. Paris 96, 379389

Stamhuis, E. J., and Videler, J. J. (1995). "Quantitative flow analysis around aquatic animals using laser sheet particle image velocimetry," J. Exp. Biol. 198, 283-294.

Webb, J. F. (1989). "Gross morphology and evolution of the mechanoreceptive lateral-line system in teleost fishes," Brain Behav. Evol. 33, 34-53.

Webb, P., Gerstner, C., and Minton, S. (1996). "Station-holding by the mottled sculpin, cottus bairdi (teleostei: cottidae), and other fishes," Copeia 2, 488-493.

Wolfgang, M. J., Anderson, J. M., Grosenbaugh, M. A., Yue, D. K. P., and Triantafyllou, M. S. (1999). "Near-body flow dynamics in swimming fish," J. Exp. Biol. 202, 2303-2327.

Wilga, C. D., and Lauder, G. V. (2001). "Functional morphology of the pectoral fins in bamboo sharks, Chilosycllium plagiosum: Benthic vs pelagic station-holding," J. Morphol. 249, 195-209.

Willert, C. E., and Gharib, M. (1991). "Digital particle imaging velocimetry," Exp. Fluids 10, 181-193. 